\title{
Performance of Arrayed Microring Resonator Sensors with the TriPleX Platform
}

\section{Invited Paper}

\section{Besselink GAJ*, Heideman RG, Schreuder E, Wevers LS, Falke F and Van den Vlekkert HH}

LioniX BV, Enschede, The Netherlands

\begin{abstract}
Integrated-optical (bio) chemical sensors are more and more commercialized, mainly because of their high intrinsic sensitivity in combination with the possibilities they offer for integration in optofluidic devices. The microring resonator (MRR), for example, is a very feasible structure to be used as microarray sensor element for multiplex biosensing. Fabrication of MRRs has been described in the case of the TriPleX platform employing well-defined stacks of stoichiometric $\mathrm{Si}_{3} \mathrm{~N}_{4}$ and $\mathrm{SiO}_{2}$ material. This platform allows for a wide variety of applications, due to its intrinsic low loss and its transparency for the VIS/NIR wavelength range. In the present paper, we describe the recent achievements in the characterization of arrays of these MRRs regarding temperature sensitivity, refractive index sensitivity and protein immobilization performance. Furthermore, the use of a reference channel/reference MRR is demonstrated in order to show the advantage of compensation of unintended change in temperature or sample composition. The refractive index sensitivity was determined to be $104 \mathrm{~nm} / \mathrm{RIU}$ and the limit of detection was about $2 \times 10^{-6}$ RIU. MRRs appeared to behave very comparable (expressed as the coefficient of (intra-array) variation $(C V))$ regarding the response to temperature $(C V \approx 0.3 \%)$ as well as refractive index $(C V<0.1 \%)$. Furthermore, it is shown that protein immobilization onto the different MRRs of the same arrays can be realized in a comparable way $(\mathrm{CV}<3 \%)$. A good comparability (i.e., a low variation) in these aspects allows for the use of internal referencing in order to compensate for unintended change in temperature or refractive index and also helps to attain a high assay precision in the ultimate biosensing application. Ongoing research is focused on the integration of more functionality on chip and further miniaturization in order to allow for fabrication of complex, though affordable, sensor devices.
\end{abstract}

Keywords: Microring resonator; MRR; Integrated optics; Refractive index; Biosensor; Sensor array

\section{Introduction}

Sensitive (bio-) chemical sensors find more and more applications in process technology, health care, environmental control, biotechnology, etc. [1-4]. Especially integrated-optical (IO) sensors are more and more commercialized (e.g., Genalyte, Optisense, PLCD, Farfield Sensors) [5], mainly because of their high intrinsic sensitivity [6] in combination with the possibilities they offer for integration in optofluidic devices [7]. Many different types of IO sensors have been described in the literature such as grating coupler devices, silicon wires, photonic crystals, waveguide interferometers and microring resonators (MRRs) [6,8,9]. Application of these sensors offers important advantages such as the prospect of label-free detection, the possibility of real-time measurement (bio-specific interaction analysis), immunity to electromagnetic interference, and the high potential for integration with other (micro) components. Furthermore, the planar waveguide devices based on silicon-on-insulator (SOI) and TriPleX platforms are fabricated in standard complementary metal-oxide semiconductor (CMOS)-compatible fabrication equipment. CMOS technology inherently offers advantages such as the potential of high precision fabrication, compactness and potential for mass production.

Further improvement in sensor sensitivity as well as advancement in integration and miniaturization is expected to add greatly to the applicability of integrated-optical systems. Due to the standardization of device and component fabrication, more and more functionality on chip becomes feasible, allowing for complex though affordable sensor devices. Current research is therefore focused on the use of multiplex sensor arrays and further miniaturization in order to reduce cost price. MRRs have an advantage because sensor signal yield and sensitivity are independent from the size of the MRR, which allows advanced miniaturization [10]. This makes the application of MRRs as a microarray sensor element for multiplex biosensing very feasible [11].
Recently, we have described the development and characteristics of a MRR sensor component in the TriPleX platform [12]. This previous paper shows the basic functionality of the TriPleX based MRR, and its applicability for lab-on-a-chip based (bio) sensing.

In the present work, arrays of TriPleX MRRs (6 sensing and 1 reference MRR per array) are described and characterized with respect to the intra-array variation between the MRRs regarding the following aspects:
1. Temperature sensitivity
2. Refractive index sensitivity
3. Amount of immobilized protein achieved by
a) Physical adsorption onto the bare waveguide surface
b) Chemical coupling onto a functionalized coating
4. Amount of analyte binding onto biosensor surface

The extent of these variations (aspects 1,2,3a and 4) is determined by the fabrication tolerance in MRR characteristics such as waveguide core layer thickness and waveguide width (see below: 'Background') and by (aspects $3 \mathrm{~b}$ and 4 ) the variability of chemical parameters such as coating thickness and density in functional groups. A good

${ }^{*}$ Corresponding author: Besselink GAJ, LioniX BV, PO Box 456, $7500 \mathrm{AL}$ Enschede, The Netherlands, Tel: +31 532030053; E-mail: g.a.j.besselink@lionixbv.nl

Received May 31, 2016; Accepted June 13, 2016; Published June 23, 2016

Citation: Besselink GAJ, Heideman RG, Schreuder E, Wevers LS, Falke F, et al. (2016) Performance of Arrayed Microring Resonator Sensors with the TriPleX Platform. J Biosens Bioelectron 7: 209. doi:10.4172/2155-6210.1000209

Copyright: @ 2016 Besselink GAJ, et al. This is an open-access article distributed under the terms of the Creative Commons Attribution License, which permits unrestricted use, distribution, and reproduction in any medium, provided the original author and source are credited. 
comparability (i.e., a low variation) in these aspects will allow for the use of internal referencing in order to compensate for changes in temperature and refractive index and will also help to attain a high assay precision. In part of the experiments the explicit use of a reference channel/reference MRR is described in order to show the advantage of compensation of the disturbing effects of unintended change in temperature or sample composition.

\section{Background}

Fabrication of integrated MRRs has been described in the case of all three main IO main platforms, especially SOI $[10,13,14]$ but also InP [15] and $\mathrm{Si}_{3} \mathrm{~N}_{4}[12,16,17]$ while realization was also reported for polymer systems $[18,19]$. The SOI platform offers a large refractive index contrast and therewith a small allowable bend radius and thus footprint. The TriPleX platform allows for a wide variety of applications, due to its intrinsic low loss $(<0.1 \mathrm{~dB} / \mathrm{cm})$ and its transparency for the wavelength region between $400 \mathrm{~nm}$ and $2.5 \mu \mathrm{m}$ [20]. TriPleX waveguides can therefore be operated with low-cost, lowpower and high-speed Vertical Cavity Surface Emitting Laser (VCSEL) light sources, as are available for the red $(\lambda \approx 650 \mathrm{~nm})$ and especially the NIR $(\lambda \approx 850 \mathrm{~nm})$ wavelength region.

Different approaches are described in the literature regarding the in- and out-coupling of light and the on-chip circuitry of MRR arrays. De Vos et al. [21] describe in-coupling of light $(\lambda \approx 1550 \mathrm{~nm})$ from a tunable laser by vertical grating coupling with connection of multiple MRRs to a common input waveguide. The signal from the individual drop ports are near-vertically coupled to free space by means of integrated vertical grating couplers and its intensity is quantified by means of an infrared camera. The resonators in series differ by $30 \mathrm{~nm}$ in circumference to avoid spectral overlap of resonances [21]. Luchansky et al. [14] also employ vertical grating couplers but with a separate input for each individual MRR and, instead of the drop port, the intensity of the through port signals is monitored by means of an infrared camera. Ghasemi et al. [16] employ end-fire coupling of tunable laser light $(\lambda \approx 655 \mathrm{~nm})$ into a common bus coupled to an array of 5 SiN MRRs. The light coming from the through port is projected on a photodetector in order to determine the resonance wavelength. Just like the approach of de Vos et al. [21] there are slight differences in the radii of the MRRs in order to prevent resonance overlap in the spectral domain.

In the case of the presently described approach, in-coupling and out-coupling of light was realized by butt-end coupling of fibers to the IO waveguides by means of an optimized fiber array (FA). The on-chip circuitry consists of a spotsize convertor (allowing for a very efficient fiber-to chip coupling efficiency, a $1 \times 8$ splitter (based on subsequent Y-branches), with each branch connected to an individual MRR, and each MRR output channel connected to a fiber through a spotsize convertor. The operating wavelength is $850 \mathrm{~nm}$, allowing the use of cheap but high quality light sources (VCSELs) and detectors (PIN photodiodes). For a detailed description of the layout of the TriPleX waveguide structures, the design of the MRR layout and the electronic interrogation scheme, please see [12].

In integrated-optical devices, thin planar waveguides are being used consisting of a stack of high refractive index core and low refractive index cladding material. In the case of TriPleX this concerns welldefined stoichiometric $\mathrm{Si}_{3} \mathrm{~N}_{4}$ and $\mathrm{SiO}_{2}$ material with a refractive index (@850 nm, TE polarized) of 1.45 and 2.0, respectively. Confinement and propagation of light can be described by the so-called total internal reflection (TIR) at the boundary between the core and the cladding. The largest part of the electromagnetic field of the light is confined to the waveguide core but a significant part propagates outside the waveguide. This component is called the evanescent field, which decays exponentially in the cladding. Measurement and simulations show a typical penetration depth of about $100 \mathrm{~nm}$, which however is very much dependent on the waveguide geometry. The MRR structure we use is a so-called racetrack resonator, which has two straight sections, the so-called directional couplers, and two semi-circular end loops (Figure 1). The directional couplers are located parallel to each of the bus waveguides over a distance $L_{D C}$ and are separated from these by a distance $g$. Light that comes in via bus waveguide 1 can be transmitted via the directional coupler to the MRR by coupling of the electric field through the evanescent tail of the light. Under non-resonance conditions, the light in the MRR will interfere in a destructive way and, consequently, most of the light that comes in via bus waveguide 1 will pass via the through port (Figure 1).

The resonance of the light waves in the MRR waveguide occurs when the wavelength fits an integer number of times $m$ in the optical path length of the MRR, the optical path length being the product of the geometric round trip length $L(=872 \mu \mathrm{m})$ and the effective refractive index $n_{\text {eff }}$ :

$$
\lambda_{\text {res }}=\frac{L^{*} n_{\text {eff }}}{m}
$$

If resonance occurs, significant part of the incident light will be transmitted via the directional couplers from bus waveguide 1 to the MRR and then to bus waveguide 2 (drop port) and this light will go to the detector. In Figure 2 an example is shown of the spectral through and drop signal response of a VCSEL/MRR combination for TE polarized light.

The MRR can be used for measurement of the bulk refractive index $n$ of the liquid that is in contact with the MRR sensor surface. Sensing of the optical properties of sample occurs in the region of a specific sensing window where the cladding is removed and the sample is in contact with the waveguide. The precise value of the resonance wavelength $\lambda_{\text {res }}$ depends on the effective refractive index of the waveguide $n_{\text {eff }}$. The bulk sensitivity $\mathrm{d} \lambda_{\text {res }} / \mathrm{dn}$ obtained from calculations and simulations using the parameters $\lambda=850 \mathrm{~nm}, \mathrm{R}=50 \mu \mathrm{m}, \mathrm{LDC}=200 \mu \mathrm{m}, \mathrm{g}=0.75 \mu \mathrm{m}, \mathrm{t}=100$ $\mathrm{nm}$, and $\mathrm{w}=1.0 \mu \mathrm{m}$, is [12]:

$$
\frac{d \lambda_{\text {res }}}{d n_{c}}=98 \mathrm{~nm} / \mathrm{RIU}
$$

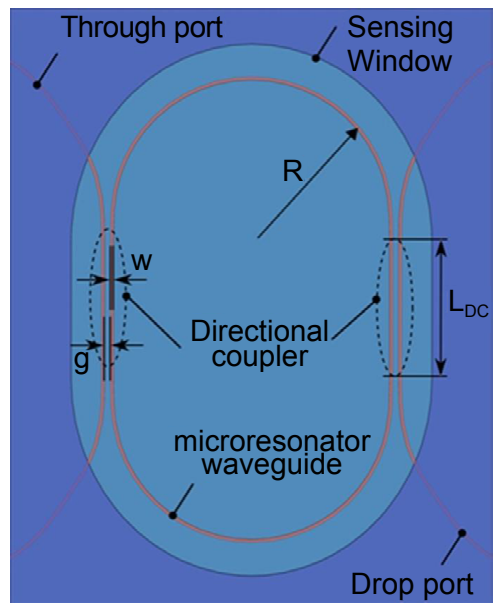

Figure 1: Schematic overview of a MRR resonator (dimensions of the current design: $R=100 \mu \mathrm{m}, \mathrm{L}_{\mathrm{DC}}=50 \mu \mathrm{m}, \mathrm{g}=0.75 \mu \mathrm{m}$ and $\mathrm{w}=1.0 \mu \mathrm{m}$ ) 


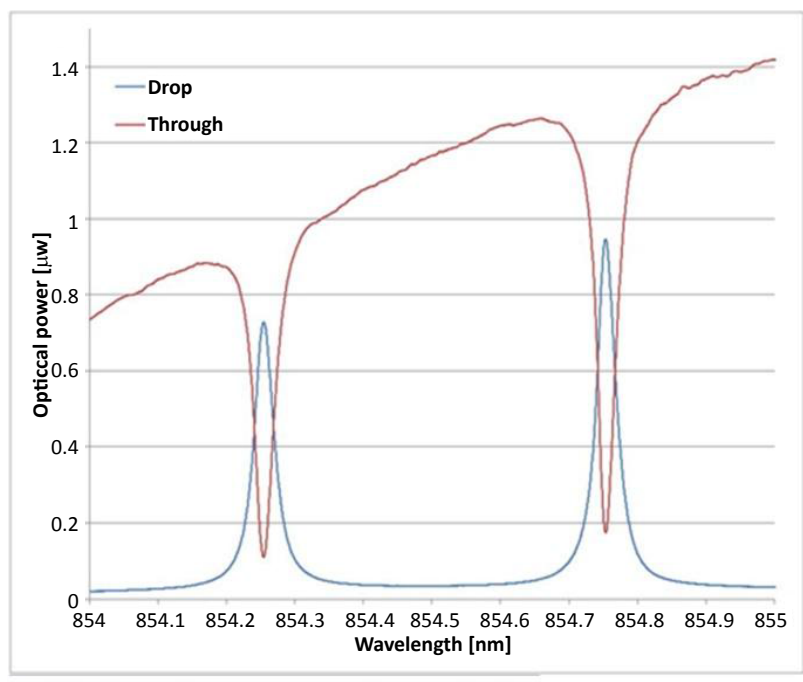

Figure 2: Resonance spectrum of a MRR resonator and VCSEL showing the drop port and through port signal, respectively.

The MRR approach is an evanescent field sensing technology so it only probes a very thin $(<<1 \mu \mathrm{m})$ layer near the MRR surface. Replacement of aqueous buffer $(\mathrm{RI} \approx 1.33$ ) by protein $(\mathrm{RI} \approx 1.45-1.55$, depending on the wavelength) at or near the MRR surface will lead to an increase of the effective refractive index of the waveguide's propagation mode. This makes the MRR suited as biosensor element for monitoring of the presence of protein (and other (bio) molecules) when these bind from the sample liquid onto the MRR surface. The used value for the refractive index of the bioreceptor layer - even though the thickness is small (below $10 \mathrm{~nm}$ ) - has a significant influence on surface sensitivity calculations. The theoretical surface sensitivity $\mathrm{d} \lambda_{\text {res }} / \mathrm{dt}_{\text {bio }}$ for the detection of protein has been calculated assuming a refractive index of 1.556 at $850 \mathrm{~nm}$ wavelength, based on Cauchy extrapolation from reported indices at lower wavelengths [12].

$$
\frac{d \lambda_{\text {res }}}{d t_{\text {bio }}}=229 \mathrm{pm} / \mathrm{nm}
$$

This surface sensitivity can also be expressed as a function of the surface concentration $\Gamma$, i.e., the adsorbed surface mass density of protein $\left(\mathrm{ng} / \mathrm{cm}^{2}\right)$. One commonly accepted value for the average mass density of proteins is $1.35 \mathrm{~g} / \mathrm{cm}^{3}$. A protein layer with a thickness of 1 $\mathrm{nm}$ is therefore roughly equivalent to a surface mass density of $135 \mathrm{ng} /$ $\mathrm{cm}^{2}$. The surface sensitivity expressed as a function of adsorbed protein mass is therefore:

$$
\frac{d \lambda_{\text {res }}}{d \Gamma}=1.7 \frac{\mathrm{pm}}{\frac{\mathrm{ng}}{\mathrm{cm}^{2}}}
$$

The sensitivity characteristics described by Equations 2-4 mainly depend on wavelength core layer thickness $t$ and waveguide width $w$ but are nearly independent of the MRR bending radius $R$, the MRR round trip length RTL, the length of the directional coupler $L_{D C}$, and the gap of the directional coupler gap $g$ (Figure 1) [12].

\section{Materials and Methods}

\section{Materials}

Bovine serum albumin (BSA) (essentially fatty acid free, $>96 \%$ ), thrombin from human plasma (lyophilized power), ethanol, Hepes,
$\mathrm{NaOH}$, 2-mercaptoethanol, phosphate buffered saline (PBS) tablets, sucrose (>99.5\%), Triscarboxy ethylphosphine (TCEP.HCl), and Triton X-100 were obtained from Sigma-Aldrich. Neutravidin (NeutrAvidin Protein) was supplied by ThermoFisher Scientific. Biotinylated BSA (No-WeighTM Biotinylated BSA) was obtained from Pierce (Rockford, IL, USA). Thiolated peptides (c-Abl substrate peptide (sequence: CEAIYAAPFAKKK) and its phosphorylated form), and monoclonal affinity-purified anti-phosphotyrosine mouse antibody were kindly provided by Dr. Rob Ruijtenbeek (University Utrecht, The Netherlands). Biotin modified DNA oligonucleotide ([BIOTEG] GGG GTT GGT GTG GTT GG), designated as thrombin binding aptamer, was obtained from Eurofins Genomics GmbH (Ebersberg, Germany).

\section{Methods}

Chip design: The design of the multi MRR chip is shown in Figure 3. The chip incorporates two moieties with each its own splitter design (based on subsequent Y-branches): one $1 \times 8$ design ( 1 input $\times 8$ sensor elements plus outputs; left half) and one $1 \times 4$ design ( 1 input $\times 4$ sensor elements plus outputs; right half) and in principle each of the designs can be used for connection to the fiber array (FA) in order to enable the in-and outcoupling of light. Such a combined design offers flexibility as, depending on the research needs or practical considerations, one can choose one of the two. Figure $3 \mathrm{C}$ shows a photomicrograph of a fabricated sensor chip.

The results of the present report relates to the $1 \times 8$ design. Corresponding biosensor contains 7 MRRs and 1 asymmetric Mach Zehnder Interferometer (a-MZI) in two variants: one $1 \times 8$ variant (Figures 3A and 3C) with all elements covered with $\mathrm{SiO}_{2}$, which was used for examining the temperature sensitivity of the MRRs. Another variant (Figure 3B) contains 6 sensing MRRs that directly contact the sample and other liquids by using a sensing window, as well as 1 MRR and $1 \mathrm{a}-\mathrm{MZI}$ that are $\mathrm{SiO}_{2}$ covered. The a-MZI is intended to be used for internal calibration of the modulated wavelength output of the VCSEL.

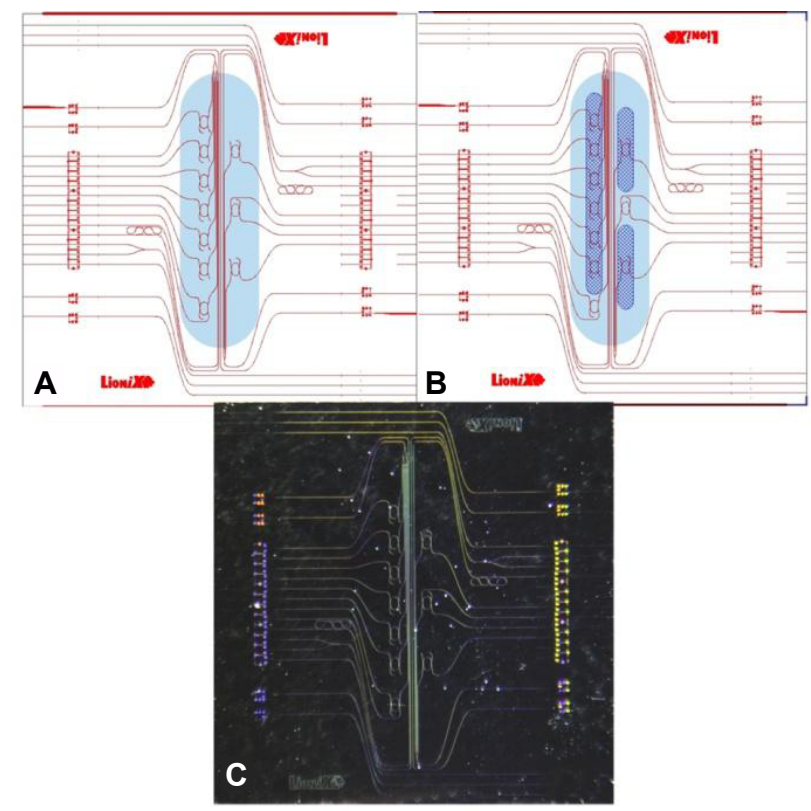

Figure 3: Design variants of the multi MRR chip. A: without, and B: with sensing windows (indicated in purple) C: Photomicrograph of the fabricated chip (without sensing window) $(1 \times 1 \mathrm{~cm})$. 
The on-chip circuitry consists of a spotsize convertor (allowing for a very efficient fiber-to chip coupling efficiency), a $1 \times 8$ splitter (based on subsequent Y-branches) with each MRR connected to a waveguide branch, and each MRR drop port connected to a fiber, again by means of a spotsize convertor. The chip is attached to an optimized fiber array (FA), which is connected to the light source (850 nm VCSEL) and detectors (PIN). The fiber-to fiber losses per channel are very small $(<3$ $\mathrm{dB})$. The assembly of chip and FA was carefully fixed by application of UV glue. For a detailed description of the layout of the waveguide structures of the MRR chip, the design of the layout and the electronic interrogation scheme, one is referred to [12] .

In part of the experiments, so-called cuvette chips ( 2 channels) were used (dimensions: $6.4 \times 2.8 \times 0.5 \mathrm{~mm}$ (lxwxh); volume $\approx 9 \mu \mathrm{l}$ ) (Figure 4). The very advantage of this type of chip is that, dictated by physical constraints, a distinction is made between the MRR that is located in one channel and the MRR that is located in the other channel. This enables one to apply selective modification of the MRRs when no mean for micro spotting is available. Furthermore, two different streams, for example a sample stream and a reference stream, can be applied during the ultimate biosensing operation. The reference MRR can be used for compensating interfering effects such as, amongst other things, fluctuations in VCSEL output and temperature induced drift. Using the differential signal is a very powerful tool as in this way successful measurement is reported on detecting small amounts $(0.005 \%)$ of ethanol in water [22]

MRR measurement platform: The MRR measurement platform consists of an optical signal read-out module (the so-called OSROM) (Figure 5), which contains a tunable light source and 8 PIN photodiode detectors, plus associated electronics and a built-in DAQ unit. As a light source, a VCSEL (type ULM850, TO46, $2.0 \mathrm{~mW}$; obtained from Philips Technology GmbH, U-L-M Photonics, Ulm, Germany) with a nominal wavelength of $850 \mathrm{~nm}$ was used. Other parts of the OSROM are a current source for driving the VCSEL diode as well as a thermal controller of the embedded VCSEL. By varying the voltage/drive current, the wavelength can be swept over a wavelength range of up to $3 \mathrm{~nm}$.

The second module of the platform is the liquid handling unit (Figure 5), which was built in-house and enables execution of automated sample injection and washing. The main part of the liquid handling system consists of two (micro) peristaltic pumps, three valves, and the associated vials and tubing. In Figure 6, a schematic illustration of the fluidic handling system is shown. Pumps and valves are operated via Lab View based liquid handling scripts that are user-defined. In the current configuration (P625/275 pumps and pump tube set P625/ TS020P (Instech Laboratories, Inc., USA)) the pump can deliver 34-340 $\mu \mathrm{L}$ per minute. The main transportation route to the chip proceeds via an 8-way valve (Figure 6: valve at the left), which enables one to switch

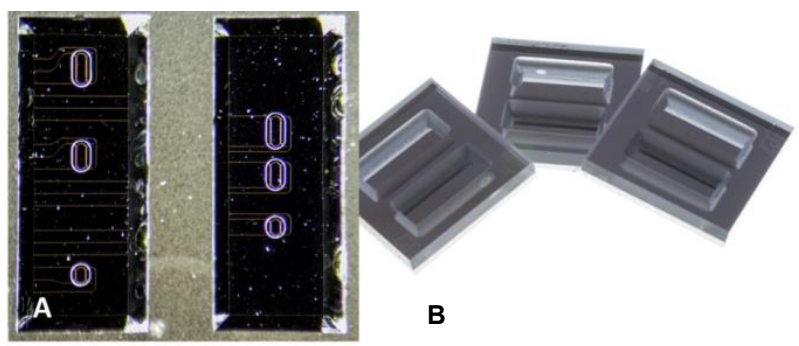

Figure 4: A: Photomicrograph of cuvette MRR chip (top view) B: View of the microcontainers.

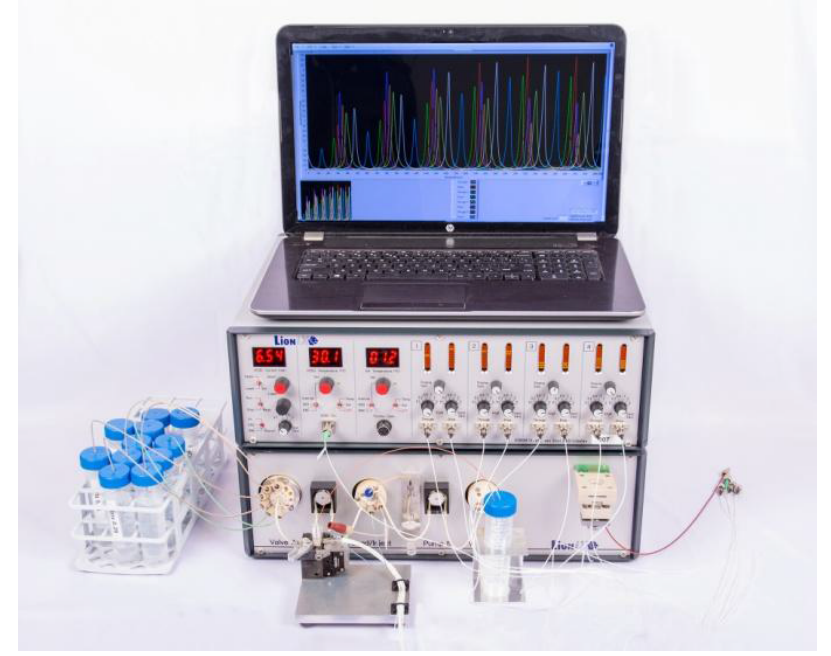

Figure 5: Photograph of the OSROM (upper instrument) and the automated liquid handling part (lower instrument) that are part of the MRR measurement platform.

from one solution to the other, such as buffer, water, cleaning solution, regeneration solution and sample. An alternative supply route to the chip proceeds via a so-called load/inject valve (Figure 6: valve at the right). This load/inject valve is supplied with a sample loop consisting of tubing of a certain capillary volume; in the present study a sample loop volume of $30 \mu \mathrm{l}$ was used. The filling of the sample loop is conducted by an independent pump and can therefore be executed apart from the main supply toward the chip. Advantage from the use of an extra pump and valve is that loading of the sample loop can be done parallel to the preconditioning of the chip. Measurement of the baseline then is done during infusion of buffer over the chip surface while the sample loop is being filled with sample. After filling the sample loop, its content $(30 \mu \mathrm{l})$ can be injected over the chip by changing the setting of the load/inject valve from load mode to inject mode. Cleaning and flushing of the sample loop and associated tubing serve to prevent contamination and carry-over between consecutive loading and measurement runs. For this purpose, an extra valve (Figure 6: valve in the middle) is connected to different vials containing cleaning solution and other liquids.

One way for incubation with sample, apart from unidirectional flow, is by using the so-called oscillating flow. In the case of oscillating flow, the center of a sample plug is brought toward the middle of the sensor by a timed pumping. Flow is stopped then after which the sample plug is repeatedly moved back and forth over the sensor by pumping alternately in one and the other direction, at a volumetric flow rate of about $0.7 \mu \mathrm{l} / \mathrm{s}$. The clear advantage of the oscillating flow approach is that, even with prolonged incubation, only a limited sample volume is needed. One disadvantage of the oscillating flow approach is that injected sample will become dispersed during the process of injection and transport toward the chip (dead volume $\approx 90 \mu \mathrm{l}$ ) caused by diffusion and convection. Consequently, this resulted in a dilution factor of about 0.5. Because of the automation of the liquid handling, the extent of dilution is expected to be reproducible and can be taken into account by choosing a more concentrated solution to start with.

Chip holder: Testing of the multi (1x8) MRR chip (Figure 3 ) was performed in combination with newly developed chip holder cartridge shown in Figure 7A. The chip holder provides a liquid-tight enclosure of the chip and is important for defining the boundary conditions of 


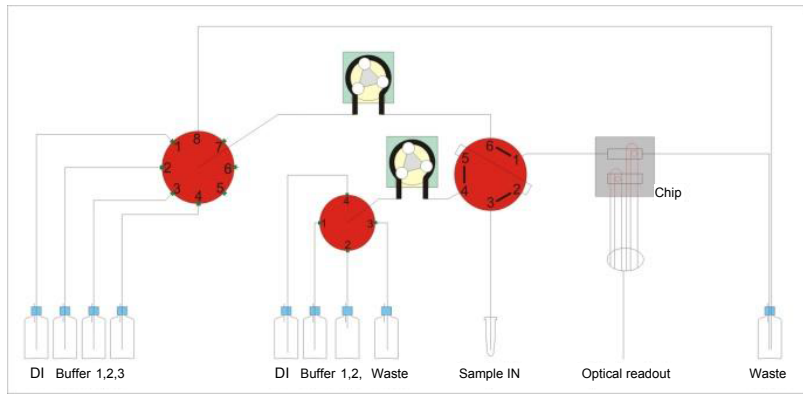

Figure 6: Schematic representation of the liquid handling system.
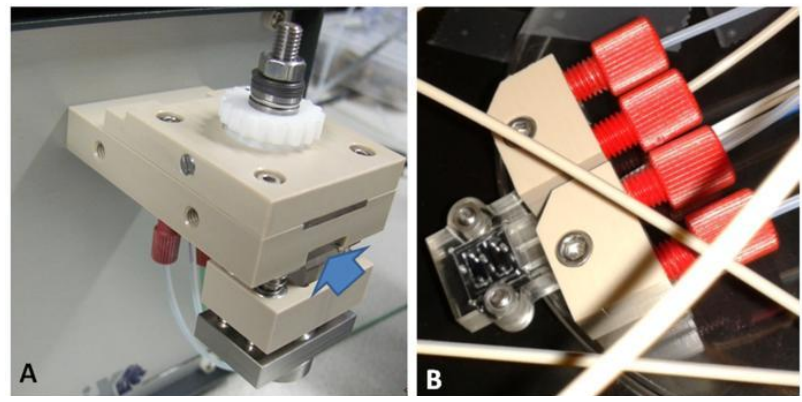

Figure 7: A: Photomicrograph of the chip holder for the $1 \times 8$ multi MRR chip place of insertion for the chip is indicated with a blue arrow B: Photomicrograph of the holder for the cuvette chip with the two-channel flow cell.

the microfluidics regime. The flow cell has an elongated shape with axe dimensions of $2 \mathrm{~mm}$ and $6 \mathrm{~mm}$ and a depth of about $100 \mu \mathrm{m}$ (cell volume $\approx 1.2 \mu \mathrm{l})$.

A second microchip holder was specially designed and fabricated for the MRR cuvette chip. The holder contains two supply channels (Figure 7B) that fit on the chip reservoirs in order to provide a continuous liquid flow over the reference channel the same way as was done for the sample channel. The chip holder was engineered and constructed by the company Epigem Ltd. (Redcar, UK) in close cooperation with LioniX.

\section{Biochemical section}

Two different approaches were used for immobilizing neutravidin protein namely passive adsorption to the bare waveguide surface and covalent immobilization to a thin sensor coating. This was done in order to examine if a more complicated chemical engineering approach (surface functionalization followed by chemical coupling) would lead to a higher intra-array variation in the amount of protein binding when compared to a simpler one-step adsorption process.

In another experiment, synthetic peptides were covalently immobilized to a maleimide functionalized sensor coating. The resulting biosensor was used in an antibody binding test to show the advantage of the use of a reference MRR. Microarrays with specific peptide sequences that may become phosphorylated are commonly used for the search and characterization of protein kinases. Phosphorylated peptides are being used as positive controls in these kinds of kinetic assays.

Neutravidin/biotinylated BSA: The preparation of a molecular capturing layer on the MRR surface was realized by physical adsorption of the neutravidin protein, which proceeded as follows: first the chips were cleaned by flushing with $0.25 \mathrm{M} \mathrm{NaOH}$. Then the chip was washed with an ample volume of MilliQ and dried by means of a stream of nitrogen gas. Next the chip was inserted into the chip holder and washed for several minutes, first with MilliQ then with PBS at a flow rate of $3 \mu \mathrm{l} / \mathrm{s}$. Subsequently, neutravidin solution $(100 \mu \mathrm{g} / \mathrm{ml}$ in PBS) was pumped over the sensor surface at a flow rate of about $0.7 \mu \mathrm{l} / \mathrm{s}$ for a total of about 10 minutes in order to allow physical adsorption of the protein. After flushing with PBS and, consecutively, PBS with $0.1 \% \mathrm{w} / \mathrm{v}$ BSA (PBS/BSA), a solution of biotinylated BSA (BSA-Biotin; $25 \mu \mathrm{g} /$ $\mathrm{ml}$ in PBS/BSA) was injected at a flow rate of about $0.7 \mu \mathrm{l} / \mathrm{s}$ for about 5 minutes followed by washing with PBS/BSA.

Neutravidin/biotinylated aptamer/thrombin: Basic functionalization of the chip $\mathrm{SiO}_{2} / \mathrm{Si}_{3} \mathrm{~N}_{4}$ surface consisted of a carboxylate layer that was applied by Surfix BV (Wageningen, The Netherlands) using their proprietary alkene technology. The following multi-step approach was followed in order to achieve immobilization of thrombin binding aptamer and to demonstrate binding of thrombin:

1. Chemical activation of the carboxylate groups by means of the EDC/NHS method (0.3 M EDC/0.1 M NHS in $10 \mathrm{mM} \mathrm{MES,}$ $\mathrm{pH}$ 5.3)

2. Coupling of the protein neutravidin $(100 \mu \mathrm{g} / \mathrm{ml}$ in MES) to the chip surface via reaction of the protein amino groups with the NHS-activated carboxylate groups

3. Capturing of biotinylated aptamer $(10 \mu \mathrm{M}$ in PBS/BSA thrombin binding aptamer) by the immobilized neutravidin

4. Binding of thrombin ( $100 \mathrm{nM}$ in PBS/BSA) to the immobilized aptamer

The preparation of the biorecognition layer on the MRR surface, i.e., the chemical coupling of neutravidin and the next step of the capturing of aptamer can be monitored using the MRR measurement. This is very helpful as it can be used as a quality control and a mean to improve the reproducibility of the performance of the resulting biosensor.

Peptide: Basic functionalization of the $\mathrm{SiO}_{2} / \mathrm{Si}_{3} \mathrm{~N}_{4}$ surface of the cuvette chips consisted of a maleimide layer that was applied by Surfix BV (Wageningen, The Netherlands) using their proprietary alkene technology. Thiolated peptides (phosphotyrosine-peptide or control peptide) were chemically coupled onto these functionalized MRR sensor surfaces. First, the thiolated peptides were pretreated with TCEP (combination of $1.5 \mu \mathrm{l}$ of $2 \mathrm{mM}$ peptide with $1.5 \mu \mathrm{l}$ of $10 \mathrm{mM}$ TCEP and $12 \mu \mathrm{l} \mathrm{MilliQ)}$ for 100 minutes at room temperature, using no stirring. This treatment for chemical reduction of disulfide bonds was performed in order to break up any dipeptide that may have been formed during storage of peptide stock solution. The presence of dipeptide has to be prevented as this may compromise the coupling yield. The two-MRR chip variant contains a micro container structure on top of the chip. This micro fabricated container structure is an integral part of the chip (Figure 4B) and may contain up to $9 \mu \mathrm{L}$ per container. The container structure was utilized for manual filling and refilling in order to achieve modification of each MRR with specific peptide as follows: each of the two cuvettes was first filled with $8 \mu \mathrm{l}$ of $100 \mathrm{mM}$ Hepes, $\mathrm{pH}$ 7.5. Then $4 \mu \mathrm{l}$ was taken out and replaced by $4 \mu \mathrm{l}$ of chemically reduced peptide solution, one cuvette received the thiolated phosphor-Tyr-peptide and the other the thiolated control peptide, and left to react for 45 minutes in a humidity chamber. After the reaction, mecaptoethanol was added at a concentration of $50 \mathrm{mM}$ and allowed to react for 10 minutes in order to block any remaining maleimide groups. Finally, the chip was washed extensively with $100 \mathrm{mM}$ Hepes buffer, $\mathrm{pH}$ 7.5. 
Citation: Besselink GAJ, Heideman RG, Schreuder E, Wevers LS, Falke F, et al. (2016) Performance of Arrayed Microring Resonator Sensors with the TriPleX Platform. J Biosens Bioelectron 7: 209. doi:10.4172/2155-6210.1000209

Page 6 of 11

Antibody binding: Using the liquid handling system, the resulting biosensor was tested for the binding and detection of antibody. After equilibration of the chip surface with binding buffer (PBS $+0.1 \%$ BSA), anti-phosphotyrosine antibody in binding buffer was injected from the sample loop and incubation was done while applying oscillating flow. Titration kinetics was applied with a serial injection of an increasing amount of antibody without intermittent regeneration. Between each two injections of antibody solution the biosensor was washed with binding buffer.

\section{Results and Discussion}

The photomicrograph in Figure 8 shows an image of the $1 \times 8$ Multi MRR chip obtained during the in-coupling of red light via a common input waveguide (indicated in the figure with $I$ ). One can distinguish the waveguide structures that distribute the light over the seven MRR and one a-MZI, and the output waveguides (indicated in Figure 8B with $O$ ). Please see Figure 3 for the corresponding overall design.

Figure 9 demonstrates the resonance spectrum, i.e., the power of the drop port signal as a function of the wavelength, as determined on
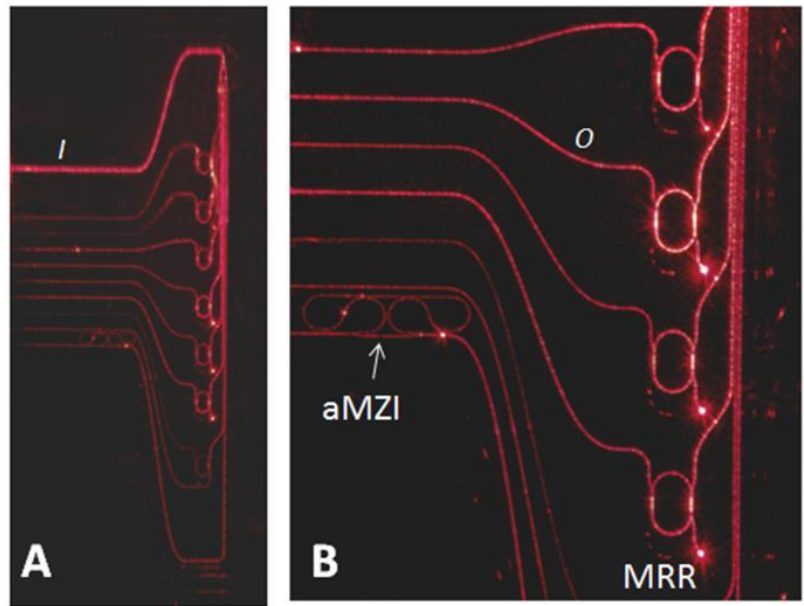

Figure 8: A: Photomicrograph of a $1 \times 8$ multi MRR chip as imaged during incoupling of light at $638 \mathrm{~nm}$ (overview) B: Zoom-in (I=common Input waveguide, $\mathrm{O}=$ Output waveguide). a multi MRR chip. Each maximum in power corresponds to a discrete resonance wavelength; each MRR has a series of succeeding resonance wavelengths. The free spectral range (FSR), the wavelength difference between each two successive maxima, typically amounts $480 \mathrm{pm}$; the full width at half maximum width (FWHM) is about $65 \mathrm{pm}$ while the Q-factor $\left(=\frac{\lambda_{\text {res }}}{F W H M}\right)$ is about $1.5 \times 10^{4}$ and the finesse $\left(=\frac{F S R}{F W H M}\right)$ is about 8 .

In a first experiment, the $\mathrm{SiO}_{2}$ covered multi MRR chip (Figure 3A) was used in order to check the intra-array comparability of the MRRs regarding the sensitivity toward temperature change. Temperature is a major determinant concerning the effective refractive index of the waveguide's propagation mode that thereby will influence the location of the resonance peaks. First a baseline was recorded and on two different times the chip was heated by means of hot air from a heat gun. As can be seen from the shift in resonance wavelength (Figure 10A) and especially from the calculated differential shift (using one of the MRRs as a reference) (Figure 10B), the MRRs behaved very comparable. Some deviation happens at the incline ramps where the slope is the steepest but apart from this there is barely any difference between the MRRs (Figure 10).

Controlled temperature stepping of the chip from $15^{\circ} \mathrm{C}$ to $50^{\circ} \mathrm{C}$ led to a shift in resonance wavelength for the seven $\mathrm{SiO}_{2}$ covered MRRs of $309 \pm 1.0 \mathrm{pm}($ mean \pm SD), which corresponds to a CV of $0.3 \%$ and a calculated temperature sensitivity of about $8.8 \mathrm{pm} /{ }^{\circ} \mathrm{C}$. Such sensitivity is rather low as compared to the value of $90 \mathrm{pm} /{ }^{\circ} \mathrm{C}$ for SOI MRR resonators as derived from the work of $\mathrm{Li}$ et al. [23]. This difference can be explained by the relatively low thermo-optic coefficient (TO) of $\mathrm{Si}_{3} \mathrm{~N}_{4}$ compared to $\mathrm{Si}$ (for comparison: $\mathrm{TO}_{\mathrm{Si3N} 4}=2.45 \times 10^{-5} \mathrm{~K}^{-1}[24]$ and $\left.\mathrm{TO}_{\mathrm{Si}}=1.87 \times 10^{-4} \mathrm{~K}^{-1}[25]\right)$. One of the structures in the biosensor Multi MRR is the oxide-covered MRR, which can be used as a reference MRR in order to assess temperature drift and fluctuations.

Next, chips were used that contained a common sensing window for six of the seven MRRs (Figure 3B) and this chip was flushed with water. Controlled temperature stepping of the chip from 15 to $40^{\circ} \mathrm{C}$ revealed a negative shift in resonance wavelength for the 6 sensing MRRs of about $36 \mathrm{pm}$, which corresponds with a calculated temperature sensitivity of about (minus) $1.4 \mathrm{pm} /{ }^{\circ} \mathrm{C}$. The difference in sign between $\mathrm{SiO}_{2}$ covered and sensing MRRs regarding temperature sensitivity is related to the

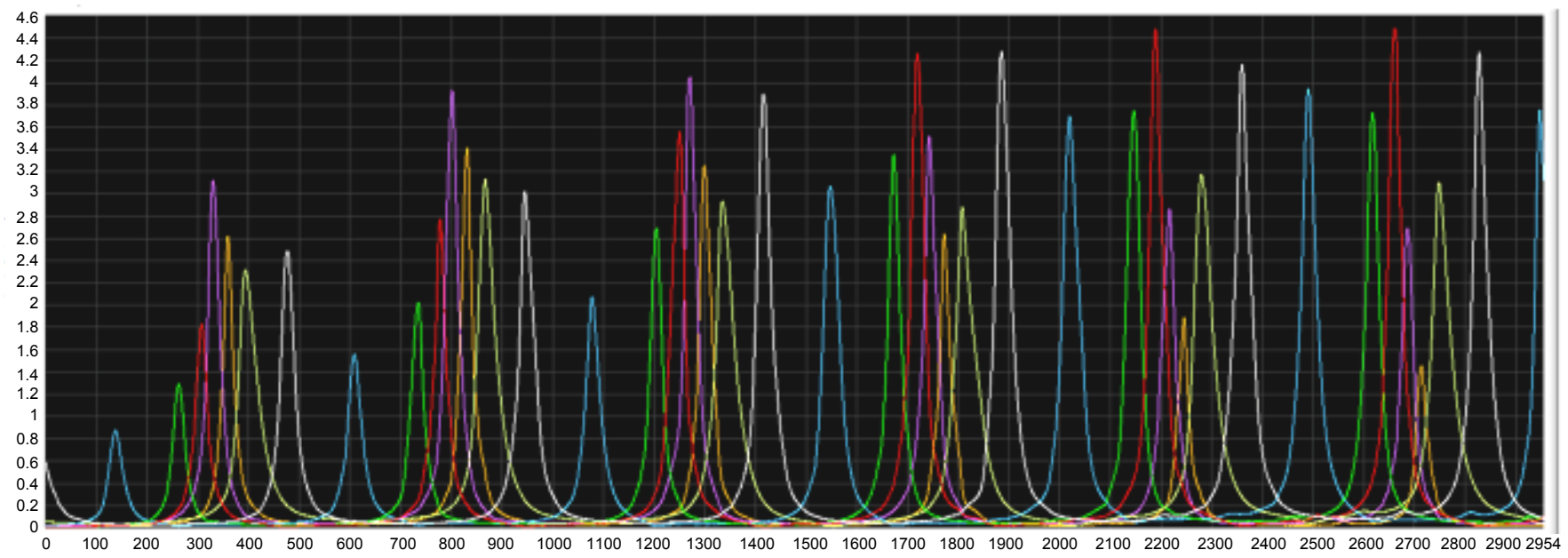

Figure 9: Example of a resonance spectrum. 


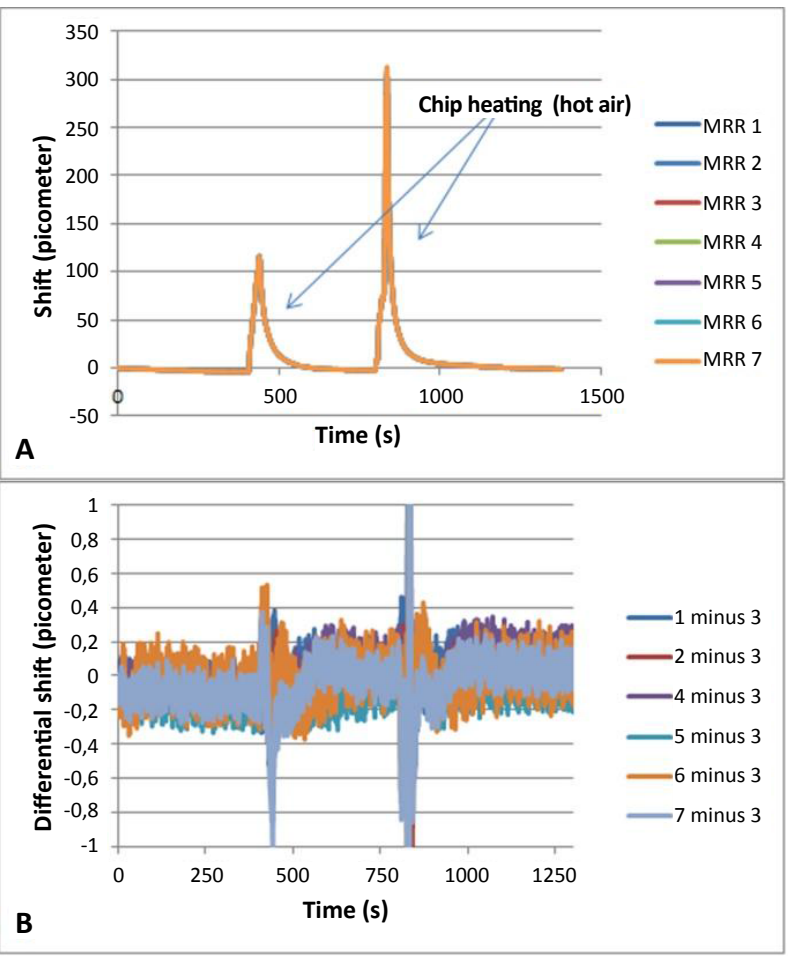

Figure 10: A: Graph showing the effect of forced heating of a multi MRR chip being the variant without sensing window (Figure 3A), on the shift in resonance wavelength B: Graph showing the calculated differential shift when one of the MRRs (\#3) was taken as a reference.

opposite sign of the thermo-optic coefficient that applies to $\mathrm{SiO}_{2}$ and water. For comparison, in the case of sensing MRRs in a SOI platform, a temperature sensitivity of $67 \mathrm{pm} /{ }^{\circ} \mathrm{C}$ is reported [26]. Unintended change in temperature is thus a less disturbing factor for TriPleX than for SOI.

The relatively small free spectral range of the TriPleX MRRs makes the tracking of a fast and large change in resonance wavelength more challenging. The OSROM measurement platform is designed for measurement rates of up to $100 \mathrm{~Hz}$ when interrogating an array of up to 8 MRRs. In practice, a measurement rate of $10 \mathrm{~Hz}$ is used. This is very fast compared to measurement rates described for other systems such as once per $8 \mathrm{~s}$ (5 MRRs [16], or 32 MRRs [14]) or once per minute (12 MRRs [21]). This measurement rate is sufficient enough to monitor drastic changes in refractive index, for example, when regeneration is carried out with high salt solutions. As an example, a fast transfer from water to $2 \mathrm{M} \mathrm{NaCl}$ induces a shift in resonance wavelength of about $2.5 \mathrm{~nm}$ in a timeframe of about $10 \mathrm{~s}$ and this can be monitored smoothly. After switching back to water the signal returns smoothly to the original baseline and this can be done very reproducible (results not shown).

In the following test, fluid was pumped over the sensor chip surface at a flow rate of $3 \mu \mathrm{l} / \mathrm{s}$ and stepping was performed between MilliQ and different solutions of sucrose. The resonance wavelength was monitored and the resulting bulk shift responses were determined as a function of the difference in bulk refractive index between the water and the different sucrose solutions. The MRRs showed very similar responses (Figure 11A) and the sensitivity for measuring refractive index was determined to be $104 \pm 0.04 \mathrm{~nm} / \mathrm{RIU}$ (mean $\pm \mathrm{SD}$ ) (Figure 11B). Measurement on different batches of chips delivers some interbatch variation in the measured sensitivity $(107 \pm 3 \mathrm{~nm} / \mathrm{RIU}$, mean \pm
$\mathrm{SD})$. This value is higher than the predicted sensitivity from Equation 2 , which is supposed to be caused by an underetching artifact of the waveguides that occurred during chip fabrication. Considering a random noise level of $0.05 \mathrm{pm}$, the theoretical detection limit $(\Delta \mathrm{n}$ at S/ $\mathrm{N}=3$ ) for measuring changes in bulk refractive index is calculated to be about $2 \times 10^{-6} \mathrm{RIU}$, which is identical to the value reported by Ghasemi et al. for SiN MRR resonators [16].

In order to verify the practical feasibility of measuring close to this detection limit and to demonstrate the advantage of the use of a reference MRR, an experiment was performed with the cuvette two-channel chip. Figure 12A shows the shift in resonance wavelength determined in case of the MRR in the channel that experiences sequential flow of two different liquids as well as the MRR in the channel with the continuous flow of reference liquid, i.e., PBS $+0.1 \%$ Tween 20 (the line in red and the line in purple, respectively). Switching was performed between PBS+0.1\% Tween 20 with and without ethanol $(0.01 \%(\mathrm{w} / \mathrm{w}))$; the corresponding difference in refractive index between the solutions was estimated to be about $6 \times 10^{-6} \mathrm{RIU}$. The signal of the sample channel revealed a repeated passage of ethanol plugs together with a significant drift of the baseline (Figure 12A). The graph of the differential shift shows a stable base level and a rather reproducible stepping signal (Figure 12B). It can be concluded that measuring a bulk refractive index difference of $6 \times 10^{-6} \mathrm{RIU}$ (and less) is achievable in the practice.

The comparability of the layer growth onto the surface of the different MRRs was examined by using a model system employing physical adsorption of neutravidin followed by capturing of biotinylated BSA. Application of a neutravidin solution onto the bare, i.e., unmodified, sensor surface for about 10 minutes led to a shift in resonance wavelength of about 500-600 pm (Figure 13). A full monolayer of neutravidin has been reported to be roughly equivalent

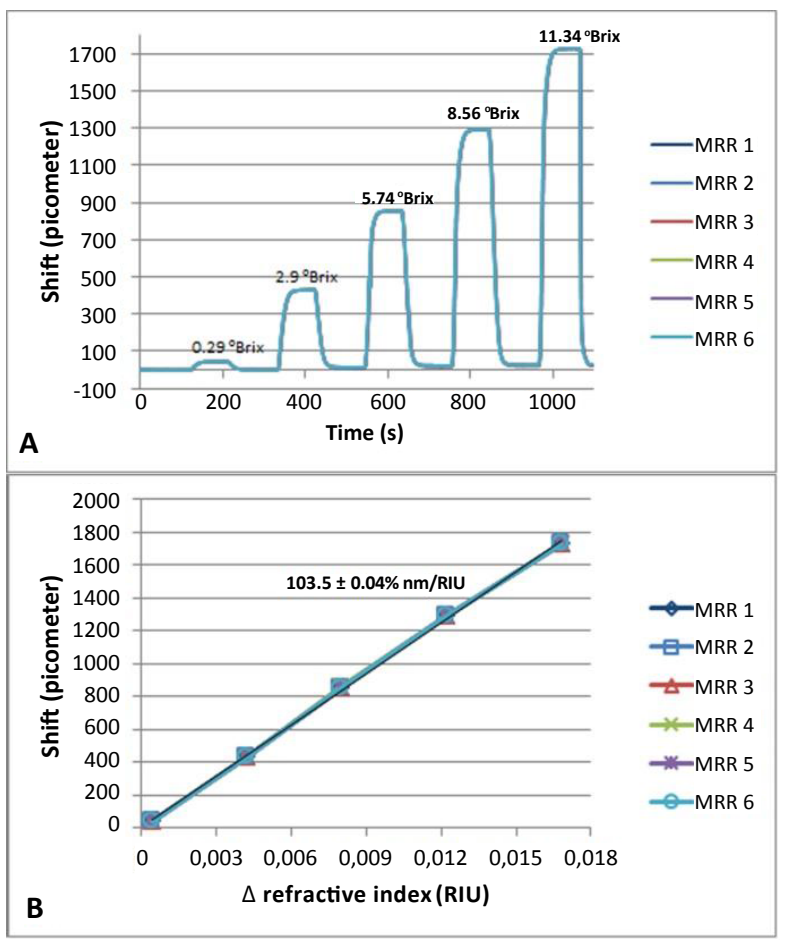

Figure 11: A: Graph showing the shift in resonance wavelength measured with a multi MRR sensor chip during the exchange of MilliQ with different sucrose solutions (concentrations indicated in ${ }^{0}$ Brix) B: Calibration lines. 
with a layer thickness of $3.5 \mathrm{~nm}$ [27], which is equivalent with a shift of about $800 \mathrm{pm}$, considering a surface sensitivity of $229 \mathrm{pm}$ shift per nm layer thickness (Equation 3). The observed shift therefore is assumed to represent about $60-75 \%$ of a packed monolayer of neutravidin.

Consecutive incubation of the resulting (neutravidin modified) sensor with biotinylated BSA and capturing of this protein onto the sensor surface led to a shift in resonance wavelength of up to about $280 \mathrm{pm}$ (Figure 13). The difference in shift observed for the first (neutravidin: $\Delta \lambda$ res $\approx 500-600 \mathrm{pm}$ ) and second layer (biotinylated BSA: $\Delta \lambda$ res $\approx 240-280 \mathrm{pm}$ ) is unexpected because, considering the similarity in molecular weight (about $60 \mathrm{kDa}$ (neutravidin) and $58 \mathrm{kDa}$ (BSA)), a near equivalent signal yield was anticipated. This dissimilarity in binding signal yield is confirmed by Altintas et al. [28] who report SPR signal yields of about 5000 and 1000 Resonance Units $(\approx 5$ and $1 \mathrm{ng} /$ $\mathrm{mm}^{2}$ of surface bound protein) for immobilization of neutravidin and capturing of biotinylated BSA, respectively. Apparently, biotinylated BSA does not pack efficiently during the binding process, which probably is caused by steric hindrance. The immobilization and binding yields that were observed for the six different MRRs were very comparable: in the experiments intra-array variations in the amount of adsorbed neutravidin and bound BSA-biotin were found in a CV range of $0.3 \%-2 \%$ from which the low-end and high-end representatives are shown in Figure 13. The different CVs are thought to be related to local heterogeneities in liquid flow within the flow cell as may be caused by, for example, the presence of air bubbles on the surfaces. Inclusion in the buffer of low concentrations of wetting agent (such as Tween 20) could be a mean to prevent this kind of interference.

In a second biochemical test, neutravidin was coupled onto a carboxylated sensor coating. The applied EDC/NHS activation method is a well-known approach for immobilization of protein, which is more

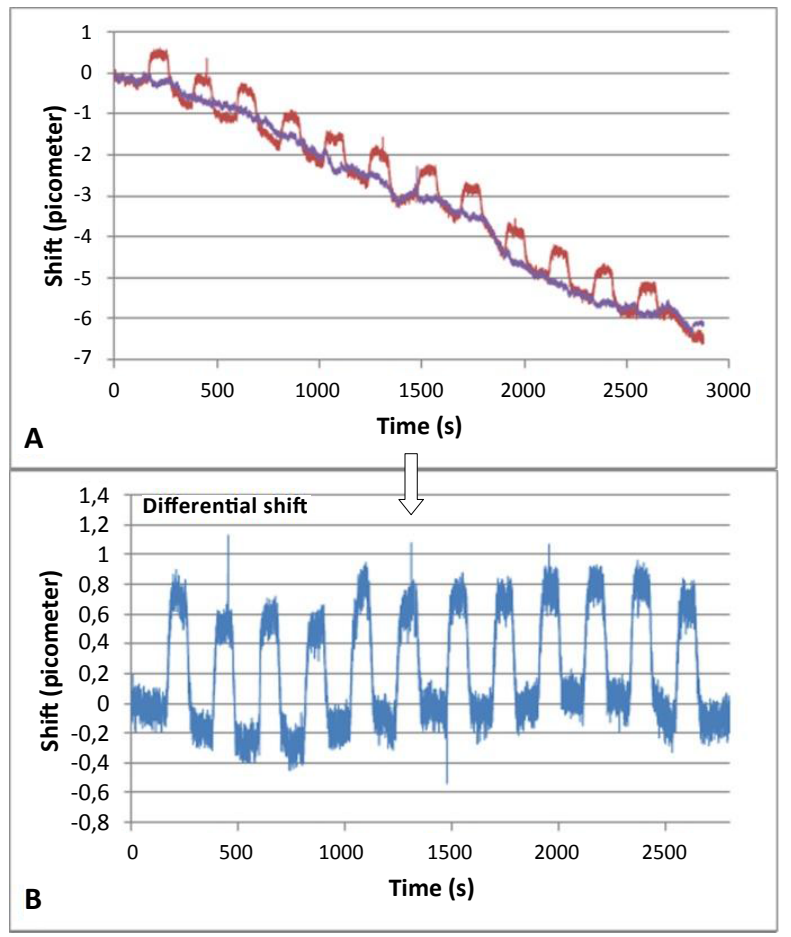

Figure 12: A: Graph showing the shift in resonance wavelength during the process of sequential exchange of buffer with and without ethanol $(0.01 \%)$ B: Graph showing the calculated differential shift. generic than the method of passive physical adsorption and yields more stable attachment. First, the intra-array variation was determined regarding the coupling yield of neutravidin onto MRRs. The binding of neutravidin onto the different MRRs proceeded in a comparable manner (Figure 14). The signal yield that resulted from covalent coupling (calculated from the shifts at $4200 \mathrm{~s}$ ) was $742 \pm 12 \mathrm{pm}$ (mean $\pm \mathrm{SD} ; \mathrm{CV}=1.6 \%)$.

We were interested if the inclusion of an extra chemical coating step and chemical coupling step would compromise the intra-array comparability of immobilized protein amount. This was not the case here as the extent of intra-array variation that was found is roughly comparable to that observed with physical adsorption onto bare sensor surface.

Next, the neutravidin modified multi MRR sensor was used for the capturing of (biotinylated) aptamer (thrombin binding aptamer). The results in Figure 15 show the comparability between the MRRs of one multi MRR chip regarding the capturing of biotinylated aptamer (left part: first $2100 \mathrm{~s}$ ). The first regeneration with $1 \mathrm{M} \mathrm{NaCl} / 10 \mathrm{mM}$ $\mathrm{NaOH}$ was done in order to prove the stable attachment of aptamer to the sensor surface. The signal that resulted from aptamer binding as determined from the shifts on $\mathrm{t}=2000 \mathrm{~s}$ was $115 \pm 3.4 \mathrm{pm}$ (mean \pm SD) (Figure 15).

Lastly, the binding of thrombin onto the aptamer was examined. Most MRR behaved very comparable during the binding phase of thrombin, with the exception of one MRR (Figure 15). The regeneration with $1 \mathrm{M} \mathrm{NaCl} / 10 \mathrm{mM} \mathrm{NaOH}$ was effective for removing the most part of the thrombin.
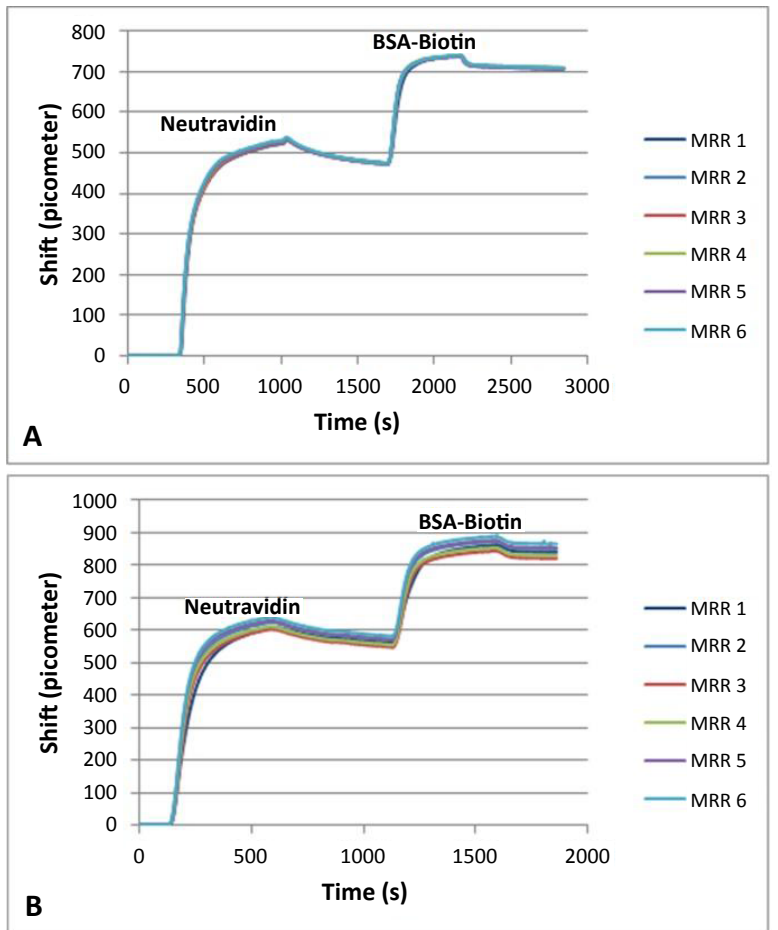

Figure 13: Sensorgrams obtained on a multi MRR sensor chip showing the shift in resonance wavelength induced by incubation with neutravidin $(100 \mu \mathrm{g} /$ $\mathrm{ml}$ in PBS) and consecutive incubation with biotinylated BSA (25 $\mu \mathrm{g} / \mathrm{ml}$ in PBS). Two examples are shown that demonstrate low $(\mathbf{A})$ and high $(\mathbf{B})$ variation. 


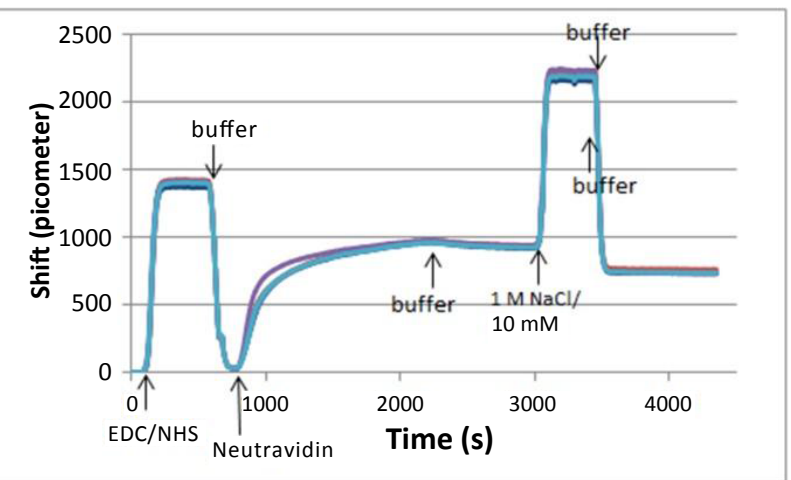

Figure 14: Sensorgram obtained with a multi MRR chip showing the whole sequence of events belonging to the chemical coupling of neutravidin onto coated sensor using the EDC/NHS method.

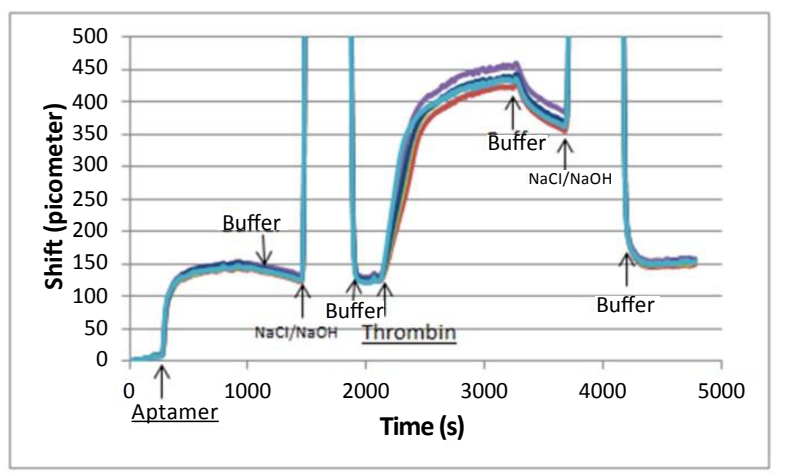

Figure 15: Sensorgram obtained with the neutravidin modified sensor showing the effect of aptamer binding, regeneration, the binding of thrombin from sample, and regeneration again.

The signal that resulted from thrombin after 15 minutes incubation time was $317 \pm 8.7 \mathrm{pm}$ (mean $\pm \mathrm{SD})$. This binding signal is comparable to the value reported by Park et al. for a SOI MRR system [13]. The intra-array variations found for aptamer capturing and thrombin binding were similar $(\mathrm{CV} \approx 2.8 \%)$.

The current practice presented in this article was in first instance dedicated to determine the degree of the intra-array variation in sensing outcome of bulk refraction index detection and layer growth monitoring. A good intra chip comparability of the MRR sensing elements is important as it will determine the ultimate level of measurement precision. In order to show the added value of a reference channel/reference $M R R$, an antibody binding experiment was performed with the cuvette chip. As a ligand, a phosphotyrosine containing peptide was used together with a reference consisting of the same but non-phosphorylated peptide sequence. Figure 16 shows the results obtained on the peptide modified two-channel chip when incubation was performed with increased amounts of anti-phosphotyrosine antibody thereby following a titration kinetics format. Measurement showed nearly no binding of antibody onto the MRR modified with control peptide (Figure 16A, curve in red). There was a significant bulk shift directly after each addition of antibody, which comes from the co-addition of glycerol, being a constituent of the antibody stock solution. The extent of the bulk shift depends from the ultimate degree of dilution of the antibody stock solution. This unintended change in refractive index can be compensated for by subtraction of the reference MRR signal. The differential signal reveals a dose-dependent binding of antibody onto the phosphotyrosine peptide that was already visible at the lowest used concentration of $0.05 \mu \mathrm{g} / \mathrm{ml}$ (Figure 16B). The specific nature of the underlying antibody interaction is further supported by the results from an extra control experiment in which a large concentration of negative control antibody $(30 \mu \mathrm{g} / \mathrm{ml}$ total rabbit $\operatorname{IgG})$ was applied and no significant binding was found (results not shown)

\section{Conclusion}

The present work shows the usability of the MRRs in the TriPleX platform for fast and sensitive real-time detection of binding events and explicitly shows the added value of using a reference MRR. Furthermore, the results are described regarding the determination of temperature sensitivity, refractive index sensitivity and protein binding characteristics on arrays of MRRs. Special consideration is given to the intra-array variation in the measured characteristics (expressed as the coefficient of variation (CV)). A temperature sensitivity of $8.8 \mathrm{pm} /{ }^{\circ} \mathrm{C}(\mathrm{CV} \approx 0.3 \%$; reference MRRs $)$ was found, and a refractive index sensitivity was demonstrated of $104 \mathrm{~nm} / \mathrm{RIU}(\mathrm{CV}<0.1 \%)$. The use of a reference MRR is therefore a good way to compensate for unintended changes in temperature and refractive index. Furthermore, immobilization of neutravidin protein and binding of biotinylated BSA, biotinylated aptamer, and consecutively thrombin could be done in an efficient way; however the corresponding CV values (0.3-3\%) were higher than the CV belonging to the measurement of temperature and RI sensitivity. Based on this, optimization of the assay precision of the ultimate biosensing application has to be directed on the improvement of the immobilization strategy and assay optimization while a possible further improvement of micro fabrication tolerances is a smaller issue.

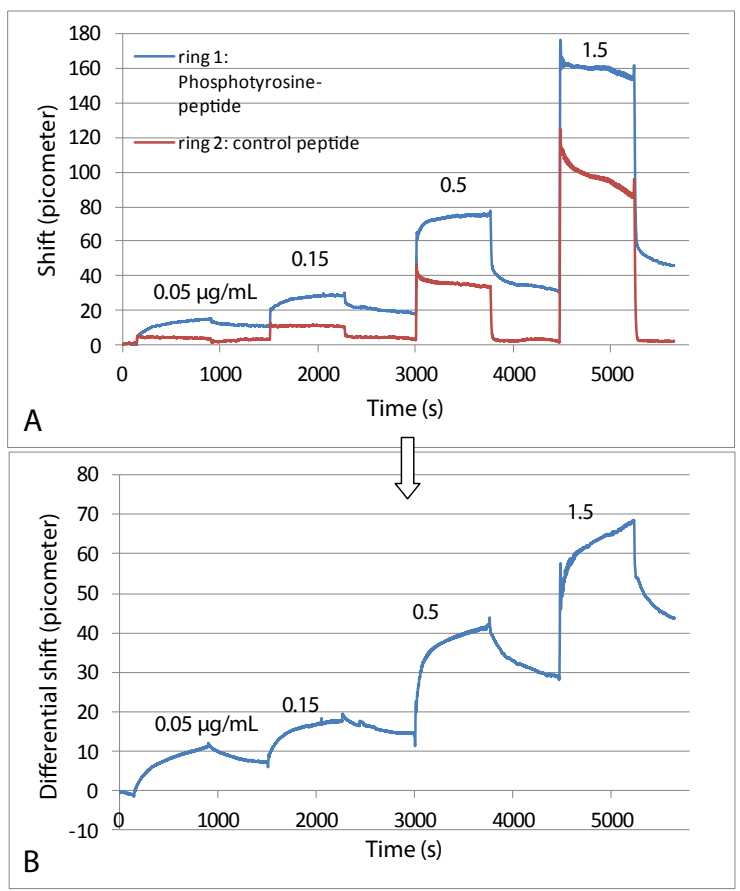

Figure 16: A: Sensorgram as determined during sequential incubation of peptide modified sensors with increasing amounts of anti-phosphotyrosine antibody with intermediate washing B: Differential shift (signal MRR minus reference $M R R$ ). 


\section{Prospect}

The presented work clearly shows state-of-the art results as obtained with IO MRRs based sensor arrays. With these sensor arrays, the light source and detectors (as well as the corresponding system driving and interrogation electronics) are positioned in a 19 inch rack, connected to the chip through stable, though expensive fiber arrays (FAs). As mentioned before, the TriPleX platform allows for the use of ultracheap though high performance light sources (VCSELS) and detectors (PIN photodiodes) operating at $850 \mathrm{~nm}$. In addition, the ultra-low loss characteristics of the TriPleX platform allows for adding additional components which can be elegantly used for on-chip calibration schemes and ("blind") compensation sensor elements.

Here, we have focused on MRRs, however also other sensor configurations can be easily combined on chip, such as the asymmetric Mach Zehnder Interferometer (a-MZI). In fact such a structure already has been implemented in the current approach in order to enable internal calibration of the VCSEL (see the a-MZI structure in Figure $8 \mathrm{~B})$. Furthermore, a-MZIs fabricated with the TriPleX platform is being used as sensor element in one of our EU projects [29,30].

Significant effort is put in hybridly combining lightsource and detectors, directly on top of the IO sensor arrays through use of grating couplers [31]. Hybrid connection of both VCSEL and PIN photodiodes has been realized through flip-chip procedure, resulting in high coupling efficiencies.

Furthermore, ongoing research is directed on the use of the multi MRR for multiplex analysis of (bio) molecules after modification of each MRR with a specific ligand by means of manual spotting or micro spotting. Generic methods for immobilization of ligand proteins onto functionalized sensor coatings will be employed. One very interesting approach is the selective modification of the $\mathrm{Si}_{3} \mathrm{~N}_{4}$ waveguide surface employing a newly developed approach from one of our consortium partners (Surfix BV).

When combined, these efforts will lead to a further miniaturization of this sensor array platform, and eventually may lead to the fully disposable disposable sensor concept in near future.

\section{Acknowledgement}

This work was supported by the EU-funded FP7 project ICT-BIOFOS (Contract No. 611528), the GO EFRO 2007-2013 programme Regio Oost-Nederland (Case No. 2011-017480), and NanoNextNL, the Dutch national research and technology programme for micro and nano technology. The authors thank Dr. Rob Ruijtenbeek (Utrecht University, Department of Medicinal Chemistry and Chemical Biology, Utrecht, The Netherlands) for his support in the peptide/antibody experiment. Furthermore, the authors thank Niko Bloemendal for his valuable work in providing the photomicrographs.

\section{References}

1. Rodriguez-Mozaz S, Alda MJ, Marco MP, Barcelo D (2005) Biosensors for environmental monitoring: A global perspective. Talanta 65: 291-297.

2. Raz SR, Haasnoot W (2011) Multiplex bioanalytical methods for food and environmental monitoring. Trends in Analytical Chemistry 30: 1526-1537.

3. Bahadir EB, Sezgintürk MK (2015) Applications of commercial biosensors in clinical, food, environmental, and biothreat/biowarfare analyses. Anal Biochem 478: $107-120$.

4. Yoo SM, Lee SY (2015) Optical biosensors for the detection of pathogenic microorganisms. Trends in Biotechnol 34: 7-25.

5. Cooper A (2006) Optical biosensors: where next and how soon? Drug Discovery Today 11: 1061-1067.

6. Fan X, White IM, Shopova SI, Zhu H, Suter JD, et al. (2008) Sensitive optical biosensors for unlabeled targets: A review. Anal Chim Acta 620: 8-26.
7. Robinson $\mathrm{G}$ (1995) The commercial development of planar optical biosensors. Sensors and Actuators B 29: 31-36.

8. Estevez MC, Alvarez M, Lechuga LM (2012) Integrated optical devices for labon-a-chip biosensing applications. Laser Photonics Rev 6: 463-487.

9. Ciminelli C, Campanella CM, Dell'Olio F, Campanella CE, Armenise MN (2013) Label-free optical resonant sensors for biochemical applications. Progress in Quantum Electronics 37: 51-107.

10. De Vos K, Bartolozzi I, Schacht E, Bienstman P, Baets R (2007) Silicon-oninsulator microring resonator for sensitive and label-free biosensing. Optics Express 15: 7610-7615.

11. Kindt JT, Bailey RC (2013) Biomolecular analysis with microring resonators: applications in multiplexed diagnostics and interaction screening. Curr Opin Chem Biol 17: 818-826.

12. Heideman R, Hoekman M, Schreuder E (2012) Triplex-based optical ring resonators for lab-on-a-chip and environmental detection. IEEE Journal of Selected Topics in Quantum Electronics 18: 1583-1596.

13. Park MK, Kee JS, Quah JY, Netto V, Song J, et al. (2013) Label-free aptamer sensor based on silicon microring resonators. Sensors and Actuators B 176 552-559.

14. Luchansky MS, Washburn AL, Martin TA, Iqbal M, Gunn LC, et al. (2010) Characterization of the evanescent field profile and bound mass sensitivity of a label-free silicon photonic microring resonator biosensing platform. Biosensors and Bioelectronics 26: 1283-1291.

15. Oh G, Lee T, Kim H, Kim D, Choi Y (2012) Design of ultra- sensitive biosensor applying surface plasmon resonance to a triangular resonator. Optics Express 20: 19067-19074.

16. Ghasemi F, Hosseini ES, Song X, Gottfried DS, Chamanzar M, et al. (2016) Multiplexed detection of lectins using integrated glycan-coated microring resonators. Biosensors and Bioelectronics 80: 682-690.

17. Muellner P, Melnik E, Koppitsch G, Kraft J, Schrank F, et al. (2015) CMOScompatible Si3N4 waveguides for optical biosensing. Procedia Engineering 120: $578-581$.

18. Chao CY, Fung W, Guo LJ (2006) Polymer microring resonators for biochemical sensing applications. IEEE Journal on Selected Topics in Quantum Electronics 12: $134-142$.

19. Xiao Y, Pichler E, Hofmann M, Bethmann K, Köhring M (2014) Towards integrated resonant and interferometric sensors in polymer films. Procedia Technology 15: 691-701.

20. Wörhoff K, Heideman RG, Leinse A, Hoekman M (2015) TriPleX: a versatile dielectric photonic platform. Adv Opt Techn 4: 189-207.

21. De Vos K, Girones J, Claes T, De Koninck Y, Popelka S, et al. (2009) Multiplexed antibody detection with an array of silicon- on-insulator microring resonators. IEEE Photonics Journal 1: 225-235.

22. Damen CAJ, Heideman RG, Heesink GJT, Schreuder F, Besselink GAJ (2014) Triplex-based microring resonators for food safety applications. Latin American Optics and Photonics Conference (LAOP), LF2D.1.

23. Li Z, Mohamed M, Chen X, Dudley E, Meng K, et al. (2012) Reliability modeling and management of nanophotonic on-chip networks. IEEE Transactions on VLSI Systems 20: 98-111.

24. Arbabi A, Goddard LL (2013) Measurements of the refractive indices and thermo-optic coefficients of Si3N4 and SiOx using microring resonances. Optics Letters 38: 3878-3881.

25. Frey BJ, Leviton DB, Madison TJ (2006) Temperature-dependent refractive index of silicon and germanium. http://cds.cern.ch/record/965045/files/0606168.pdf

26. Abdulla SMC, De Boer BM, Pozo JM, Van den Berg JH, Abutan A, et al. (2014) Sensing platform based on micro-ring resonator and on-chip reference sensors in SOI. Proc of SPIE 8990: 89900.

27. Sciacca B, François A, Klingler-Hoffmann M, Brazzatti J, Penno M, et al. (2013) Radiative-surface Plasmon resonance for the detection of apolipoprotein $E$ in medical diagnostics applications. Nanomedicine: Nanotechnology, Biology, and Medicine 9: 550-557.

28. Altintas Z, Uludag Y, Gurbuz Y, Tothill I (2012) Development of surface 
Citation: Besselink GAJ, Heideman RG, Schreuder E, Wevers LS, Falke F, et al. (2016) Performance of Arrayed Microring Resonator Sensors with the TriPleX Platform. J Biosens Bioelectron 7: 209. doi:10.4172/2155-6210.1000209

chemistry for SPR based sensors for the detection of proteins and DNA molecules. Analytica Chimica Acta 712: 138-144

29. Chalyan T, Pasquardini L, Falke F, Zanetti M, Guider R, et al. (2016) Biosensors based on Si3N4 asymmetric Mach-Zehnder interferometers. Proc of SPIE 9899: 98991S.

30. Chalyan T, Guider R, Pasquardini L, Zanetti M, Falke F, et al. (2016)
Asymmetric Mach-Zehnder interferometer based biosensors for aflatoxin M1 detection. Biosensors 6: 1.

31. Gounaridis L, Groumas P, Schreuder E, Heideman R, Katopodis V, et al (2015) Design of grating couplers and MMI couplers on the TriPleX platform enabling ultra-compact photonic-based biosensors. Sensors and Actuators B 209: 1057-1063. 